\title{
The role of three-gluon correlation functions in the single spin asymmetry
}

\author{
Hiroo Beppu ${ }^{1, a}$, Koichi Kanazawa ${ }^{1,2, b}$, Yuji Koike ${ }^{3, c}$, and Shinsuke Yoshida ${ }^{4,5, d}$ \\ ${ }^{1}$ Graduate School of Science and Technology, Niigata University, Ikarashi, Niigata 950-2181, Japan \\ ${ }^{2}$ Department of Physics, Barton Hall, Temple University, Philadelphia, Pennsylvania 19122, USA \\ ${ }^{3}$ Department of Physics, Niigata University, Ikarashi, Niigata 950-2181, Japan \\ ${ }^{4}$ Theoretical Research Division, Nishina Center, RIKEN, Wako 351-0198, Japan \\ ${ }^{5}$ Physics Department, Brookhaven National Laboratory, Upton, NY 11973, USA
}

\begin{abstract}
We study the twist-3 three-gluon contribution to the single spin asymmetry in the light-hadron production in pp collision in the framework of the collinear factorization. We derive the corresponding cross section formula in the leading order with respect to the QCD coupling constant. We also present a numerical calculation of the asymmetry at the RHIC energy, using a model for the three-gluon correlation functions suggested by the asymmetry for the $D$-meson production at RHIC. We found that the asymmetries for the light-hadron and the jet productions are very useful to constrain the magnitude and form of the correlation functions. Since the three-gluon correlation functions shift the asymmetry for all kinds of hadrons in the same direction, it is unlikely that they become a main source of the asymmetry.
\end{abstract}

\section{Introduction}

The origin of the transverse single-spin asymmetry (SSA) $A_{N} \equiv\left(\sigma^{\uparrow}-\sigma^{\downarrow}\right) /\left(\sigma^{\uparrow}+\sigma^{\downarrow}\right)$ for $p^{\uparrow} p \rightarrow h X(h=\pi, K, \eta$, jet $)$ observed by the Relativistic Heavy Ion collider (RHIC) at the Brookhaven National Laboratory (BNL) [1]-[9] is yet to be clarified in terms of the twist- 3 mechanism in the framework of the collinear factorization. This process receives, in principle, four types of the twist- 3 contributions depending on the type of the twist- 3 correlation function participating in the cross section; i.e., (i) twist-3 quarkgluon correlation function in the polarized nucleon [10][16], (ii) twist-3 three-gluon correlation function in the polarized nucleon [17]-[22], (iii) twist-3 fragmentation for the final hadron [24]-[27] and (iv) twist-3 quark-gluon correlation function in the initial unpolarized nucleon [28]. The last contribution (iv) was shown to be negligible due to the small partonic hard cross sections [29]. So far most of the analyses of $A_{N}$ for the light-hadron production has been performed, assuming that the contribution (i) is the sole origin of the asymmetry [14, 30-32]. However, the resulting quark-gluon correlation functions turned out to have a sign opposite to that of the Sivers function obtained from the analyses of SSAs observed in semi-inclusive deep inelastic scattering (SIDIS) [33]. Clearly the other sources, (ii) and (iii), should also play an important role for the asymmetry. More recently the cross section formula for (iii) was derived in [27], and an analysis of $A_{N}$ for the pion

\footnotetext{
a e-mail: beppu@nt.sc.niigata-u.ac.jp

be-mail: koichi.kanazawa@temple.edu

ce-mail: koike@nt.sc.niigata-u.ac.jp

de-mail: shinsuke.yoshida@ riken.jp
}

production at RHIC has been performed including the effects of (i) and (iii), in which the Sivers function and the transversity distribution for the nucleon and the Collins functions for the pion have been fixed using the data of SIDIS and $e^{+} e^{-}$-annihilations and the twist- 3 fragmentation function has been fitted to reproduce the RHIC $A_{N}$ data [34]. The analysis has shown that the combination can describe the $A_{N}$ data consistently with other two processes. To justify this scenario for $A_{N}$, it is also important to analyze the role of the above (ii).

In our recent papers, we have derived the contribution of the twist- 3 three-gluon correlation functions in the transversely polarized nucleon to the single spin asymmetry for the $D$-meson productions in SIDIS, $e p^{\uparrow} \rightarrow$ $e D X[19,35]$, and the pp collision, $p^{\uparrow} p \rightarrow D X[21]$, and the Drell-Yan/direct-photon processes, $p^{\uparrow} p \rightarrow \gamma^{(*)} X$ [22]. Using some models for the three-gluon correlation functions, we also studied its impact on the corresponding asymmetries at the energy of RHIC and the Electron-IonCollider (EIC). In a recent paper [23], we have extended these analyses to the light-hadron production in the pp collision. This has completed the corresponding leading order twist- 3 cross section for $p^{\uparrow} p \rightarrow h X$ together with the known result for the above contributions (i) and (iii). This talk is a summary of [23].

This paper is organized as follows: After introducing the three-gluon correlation functions in section 2, we present the twist-3 cross section in section 3. In section 4, we study its impact on the SSA in the light-hadron productions at the RHIC energy by using the model in [21, 22]. We will see the three-gluon correlation disturbs the observed patterns of the asymmetry seriously, which indi- 
cates that this process may be used to constrain the magnitude and the form of the three-gluon correlations.

\section{Three-gluon correlation functions in the transversely polarized nucleon}

As clarified in [17-19], there are two independent threegluon correlation functions in the transversely polarized nucleon, $O\left(x_{1}, x_{2}\right)$ and $N\left(x_{1}, x_{2}\right)$, which are the Lorentzscalar functions of the longitudinal momentum fractions $x_{1}$ and $x_{2}$, defined as

$$
\begin{aligned}
O^{\alpha \beta \gamma}\left(x_{1}, x_{2}\right)= & -g(i)^{3} \int \frac{d \lambda}{2 \pi} \int \frac{d \mu}{2 \pi} e^{i \lambda x_{1}} e^{i \mu\left(x_{2}-x_{1}\right)} \\
\times & \left\langle p S_{\perp}\left|d_{b c a} F_{b}^{\beta n}(0) F_{c}^{\gamma n}(\mu n) F_{a}^{\alpha n}(\lambda n)\right| p S_{\perp}\right\rangle \\
= & 2 i M_{N}\left[O\left(x_{1}, x_{2}\right) g^{\alpha \beta} \epsilon^{\gamma p n S_{\perp}}\right. \\
& +O\left(x_{2}, x_{2}-x_{1}\right) g^{\beta \gamma} \epsilon^{\alpha p n S_{\perp}} \\
& \left.+O\left(x_{1}, x_{1}-x_{2}\right) g^{\gamma \alpha} \epsilon^{\beta p n S_{\perp}}\right], \\
N^{\alpha \beta \gamma}\left(x_{1}, x_{2}\right)= & -g(i)^{3} \int \frac{d \lambda}{2 \pi} \int \frac{d \mu}{2 \pi} e^{i \lambda x_{1}} e^{i \mu\left(x_{2}-x_{1}\right)} \\
\times & \left\langle p S_{\perp}\left|i f_{b c a} F_{b}^{\beta n}(0) F_{c}^{\gamma n}(\mu n) F_{a}^{\alpha n}(\lambda n)\right| p S_{\perp}\right\rangle \\
= & 2 i M_{N}\left[N\left(x_{1}, x_{2}\right) g^{\alpha \beta} \epsilon^{\gamma p n S_{\perp}}\right. \\
& -N\left(x_{2}, x_{2}-x_{1}\right) g^{\beta \gamma} \epsilon^{\alpha p n S_{\perp}} \\
& \left.-N\left(x_{1}, x_{1}-x_{2}\right) g^{\gamma \alpha} e^{\beta p n S_{\perp}}\right]
\end{aligned}
$$

where $F_{a}^{\alpha \beta} \equiv \partial^{\alpha} A_{a}^{\beta}-\partial^{\beta} A_{a}^{\alpha}+g f_{a b c} A_{b}^{\alpha} A_{c}^{\beta}$ is the gluon's field strength, and we used the notation $F_{a}^{\alpha n} \equiv F_{a}^{\alpha \beta} n_{\beta}$ and $\epsilon^{\alpha p n S_{\perp}} \equiv \epsilon^{\alpha \mu \nu \lambda} p_{\mu} n_{\nu} S_{\perp \lambda}$ with the convention $\epsilon_{0123}=1$. $d^{b c a}$ and $f^{b c a}$ are the symmetric and anti-symmetric structure constants of the color SU(3) group, and we have suppressed the gauge-link operators which ensure the gauge invariance. $p$ is the nucleon momentum, and $S_{\perp}$ is the transverse spin vector of the nucleon normalized as $S_{\perp}^{2}=$ -1 . In the twist-3 accuracy, $p$ can be regarded as lightlike $\left(p^{2}=0\right)$, and $n$ is another lightlike vector satisfying $p \cdot n=1$. To be specific, we set $p^{\mu}=\left(p^{+}, 0, \mathbf{0}_{\perp}\right)$, $n^{\mu}=\left(0, n^{-}, \mathbf{0}_{\perp}\right)$, and $S_{\perp}^{\mu}=\left(0,0, \mathbf{S}_{\perp}\right)$. The nucleon mass $M_{N}$ is introduced to define $O\left(x_{1}, x_{2}\right)$ and $N\left(x_{1}, x_{2}\right)$ dimensionless. The decomposition (1) and (2) takes into account all the constraints from hermiticity, invariance under the parity- and time-reversal transformations and the permutation symmetry among the participating three gluon-fields. The functions $O\left(x_{1}, x_{2}\right)$ and $N\left(x_{1}, x_{2}\right)$ are real and have the following symmetry properties,

$$
\begin{array}{ll}
O\left(x_{1}, x_{2}\right)=O\left(x_{2}, x_{1}\right), & O\left(x_{1}, x_{2}\right)=O\left(-x_{1},-x_{2}\right), \\
N\left(x_{1}, x_{2}\right)=N\left(x_{2}, x_{1}\right), & N\left(x_{1}, x_{2}\right)=-N\left(-x_{1},-x_{2}\right) .
\end{array}
$$

The functions $N\left(x_{1}, x_{2}\right)$ and $O\left(x_{1}, x_{2}\right)$ are, respectively, even and odd under charge conjugation.

\section{Twist-3 cross section for $p^{\uparrow} p \rightarrow h X$ induced by the three-gluon correlation functions}

The twist- 3 single-spin-dependent cross section for the process $p^{\uparrow}\left(p, S_{\perp}\right)+p\left(p^{\prime}\right) \rightarrow h\left(P_{h}\right)+X$ induced by the three-gluon correlation functions has been obtained in [23] by applying the formalism developed in [19-22]. It reads

$$
\begin{aligned}
& E_{P_{h}} \frac{d^{3} \Delta \sigma}{d^{3} P_{h}}=\frac{2 \pi M_{N} \alpha_{s}^{2}}{S} \epsilon^{P_{h} p n S_{\perp}} \\
\times & \sum_{i, j} \int \frac{d x}{x} \int \frac{d x^{\prime}}{x^{\prime}} f_{i}\left(x^{\prime}\right) \int \frac{d z}{z^{2}} D_{j}(z) \delta(\hat{s}+\hat{t}+\hat{u}) \frac{1}{z \hat{u}} \\
\times & {\left[\zeta_{i j}\left(\frac{d}{d x} O(x)-\frac{2 O(x)}{x}\right) \hat{\sigma}_{g i \rightarrow j}^{(O)}+\left(\frac{d}{d x} N(x)-\frac{2 N(x)}{x}\right) \hat{\sigma}_{g i \rightarrow j}^{(N)}\right], }
\end{aligned}
$$

where the functions $O(x)$ and $N(x)$ are defined as

$$
\begin{aligned}
& O(x) \equiv O(x, x)+O(x, 0), \\
& N(x) \equiv N(x, x)-N(x, 0),
\end{aligned}
$$

and the factor $\zeta_{i j}$ is defined so that $\zeta_{i j}=-1$ when $i$ or $j$ is an anti-quark flavor and $\zeta_{i j}=1$ for other cases. $f_{i}\left(x^{\prime}\right)$ and $D_{i}(z)$ are, respectively, unpolarized distribution and fragmentation functions for the quark and anti-quark flavors and the gluon $(i=q, \bar{q}, g)$. The partonic hard crosss sections $\hat{\sigma}_{g i \rightarrow j}^{(O, N)}$ can be written as the sum of the contributions from the initial-state-interaction (ISI) and the final-stateinteraction (FSI) diagrams as

$$
\hat{\sigma}_{g i \rightarrow j}^{(O, N)}=\hat{\sigma}_{g i \rightarrow j}^{(O, N) I}-\frac{\hat{s}}{\hat{t}} \hat{\sigma}_{g i \rightarrow j}^{(O, N) F},
$$

and they are given in terms of the partonic Mandelstam variables, $\hat{s}=\left(x p+x^{\prime} p^{\prime}\right)^{2}, \hat{t}=\left(x p-p_{c}\right)^{2}, \hat{u}=\left(x^{\prime} p^{\prime}-p_{c}\right)^{2}$. The cross sections $\hat{\sigma}_{g i \rightarrow j}^{(O, N) I, F}$ are given as follows [23]:

(i) Unpolarized quark distribution channels:

(a) Quark fragmentation channel:

$$
\begin{aligned}
& \hat{\sigma}_{g q \rightarrow q}^{(O) I}=-\hat{\sigma}_{g q \rightarrow q}^{(N) I}=\frac{\hat{t}\left(\hat{s}^{2}+\hat{t}^{2}\right)}{\hat{s} \hat{u}^{2}}-\frac{1}{N^{2}}\left(\frac{\hat{s}}{\hat{t}}+\frac{\hat{t}}{\hat{s}}\right), \\
& \hat{\sigma}_{g q \rightarrow q}^{(O) F}=\hat{\sigma}_{g q \rightarrow q}^{(N) F}=-\frac{\hat{s}\left(\hat{s}^{2}+\hat{t}^{2}\right)}{\hat{t} \hat{u}^{2}}+\frac{1}{N^{2}}\left(\frac{\hat{s}}{\hat{t}}+\frac{\hat{t}}{\hat{s}}\right) .
\end{aligned}
$$

(b) Gluon fragmentation channel:

$$
\begin{aligned}
\hat{\sigma}_{g q \rightarrow g}^{(O) I} & =-\hat{\sigma}_{g q \rightarrow g}^{(N) I} \\
& =\frac{\hat{u}\left(\hat{s}^{2}+\hat{u}^{2}\right)}{\hat{s}^{2}}-\frac{1}{N^{2}}\left(\frac{\hat{s}}{\hat{u}}+\frac{\hat{u}}{\hat{s}}\right), \\
\hat{\sigma}_{g q \rightarrow g}^{(O) F} & =\frac{(\hat{s}-\hat{u})\left(\hat{s}^{2}+\hat{u}^{2}\right)}{\hat{s} \hat{t} \hat{u}}, \\
\hat{\sigma}_{g q \rightarrow g}^{(N) F} & =\frac{\left(\hat{s}^{2}+\hat{u}^{2}\right)^{2}}{\hat{s} \hat{t}^{2} \hat{u}},
\end{aligned}
$$

(ii) Unpolarized gluon distribution channels:

(a) Quark fragmentation channel:

$$
\begin{aligned}
\hat{\sigma}_{g g \rightarrow q}^{(O) I} & =\frac{1}{C_{F}} \frac{(\hat{u}-\hat{t})\left(\hat{t}^{2}+\hat{u}^{2}\right)}{2 \hat{s} \hat{t} \hat{u}}, \\
\hat{\sigma}_{g g \rightarrow q}^{(N) I} & =\frac{1}{C_{F}} \frac{\left(\hat{t}^{2}+\hat{u}^{2}\right)^{2}}{2 \hat{s}^{2} \hat{t} \hat{u}}, \\
\hat{\sigma}_{g g \rightarrow q}^{(O) F} & =\hat{\sigma}_{g g \rightarrow q}^{(N) F} \\
& =\left(-\frac{1}{C_{F}} \frac{\hat{u}}{2 \hat{s}^{2} \hat{t}}+\frac{1}{N^{2} C_{F}} \frac{1}{2 \hat{t} \hat{u}}\right)\left(\hat{t}^{2}+\hat{u}^{2}\right) .
\end{aligned}
$$


(b) Gluon fragmentation channel:

$$
\begin{aligned}
& \hat{\sigma}_{g g \rightarrow g}^{(O) I, F}=0, \\
& \hat{\sigma}_{g g \rightarrow g}^{(N) I}=\frac{N}{C_{F}} \frac{2\left(\hat{t}^{2}+\hat{u}^{2}\right)\left(\hat{t}^{2}+\hat{t} \hat{u}+\hat{u}^{2}\right)^{2}}{\hat{s}^{2} \hat{t}^{2} \hat{u}^{2}}, \\
& \hat{\sigma}_{g g \rightarrow g}^{(N) F}=-\frac{N}{C_{F}} \frac{2\left(\hat{t}^{2}+2 \hat{t} \hat{u}+2 \hat{u}^{2}\right)\left(\hat{t}^{2}+\hat{t} \hat{u}+\hat{u}^{2}\right)^{2}}{\hat{s}^{2} \hat{t}^{2} \hat{u}^{2}} .
\end{aligned}
$$

A remarkable feature of (5) is that the partonic hard cross sections for $O(x, x)$ and $O(x, 0)$ are identical, and likewise for $N(x, x)$ and $-N(x, 0)$. Therefore they contribute to the cross section through the combinations $O(x)$ and $N(x)$ in (6). The same feature was also observed for the twist-3 cross section for the prompt-photon production $p^{\uparrow} p \rightarrow \gamma X$. Note that (13), (14) and (15) are obtained from the result for $p^{\uparrow} p \rightarrow D X$ in [21] by taking the massless limit of the charm-quark mass, $m_{c} \rightarrow 0$. For the processes in which all particles participating in the scattering are massless, three-gluon correlations contribute in the combination of $O(x)$ and $N(x)$. This is in contrast to the case for SIDIS and Drell-Yan processes, where the virtual photon with large $Q^{2}$ enters the scattering. The result $\hat{\sigma}_{g g \rightarrow g}^{(O) I, F}=0$ in (16) is due the vanishing color factors.

\section{Numerical calculation of the asymmetry at the RHIC energy}

To illustrate the effect of the three-gluon correlation functions to $A_{N}$ for the light-hadron production, we perform here a model calculation of $A_{N}$ at the RHIC energy. In $[21,22]$, we employed two models for the three-gluon correlation in the study of $A_{N}$ in $p^{\uparrow} p \rightarrow D X$ and $p^{\uparrow} p \rightarrow \gamma X$. We use the same models here, i.e,

$$
\begin{array}{ll}
\text { Model 1: } & O(x)=N(x)=0.004 x G(x), \\
\text { Model 2: } & O(x)=N(x)=0.001 \sqrt{x} G(x),
\end{array}
$$

where $G(x)$ is the twist- 2 unpolarized gluon density. ${ }^{1}$ The coefficients 0.004 and 0.001 were determined so that the calculated $A_{N}^{D}$ does not exceed the RHIC preliminary data for $A_{N}^{D}[36]$. The above model ansatz were motivated to see the effect of the three-gluon correlations in comparison with the gluon density and to see the sensitivity of $A_{N}$ to the small- $x$ behavior of the functions. We use the unpolarized parton density in [37] and the fragmentation function for the pion in [38]. For the calculation, we set the scale of all the distribution and fragmentation functions at the transverse momentum of the final hadron $P_{T}$.

Figure 1 shows the $x_{F}$-dependence of the three-gluon contribution to $A_{N}$ for the $\pi^{ \pm, 0}$ and jet productions in the pp-collision at the RHIC energy $\sqrt{S}=200 \mathrm{GeV}$ and $P_{T}=2 \mathrm{GeV}$. We plotted the contribution from $O(x)$ and $N(x)$ separately to see each effect on $A_{N}$. At $x_{F}>0, N(x)$ gives rise to the large asymmetry and the effect of $O(x)$ is negligible for both models. The origin of the large asymmetry from $N(x)$ is the partonic cross section (18): At large $x_{F}>0$, where $-T \ll S \sim-U$, large- $x$ and small- $x^{\prime}$ region

\footnotetext{
${ }^{1}$ In this rough estimate of the three-gluon contribution to the asymmetry, we set the scale dependence of the three-gluon correlation to be the same as the twist-2 gluon density.
}
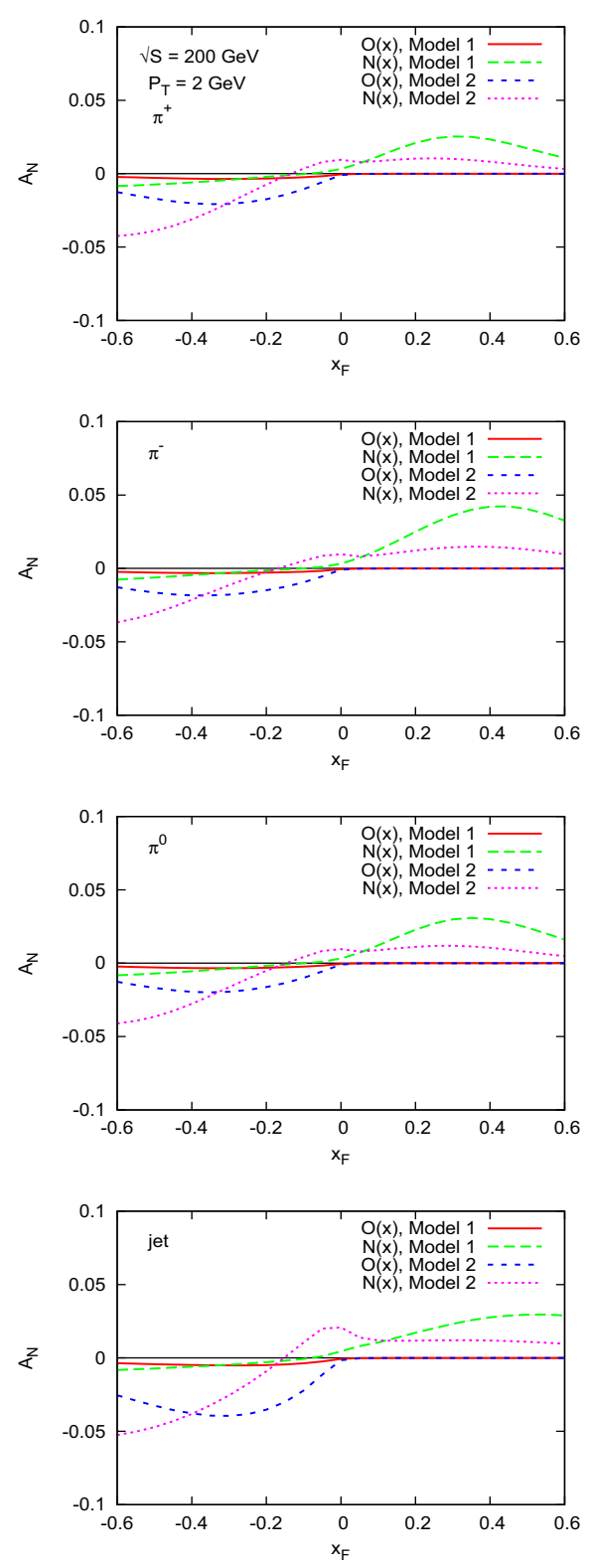

Figure 1. $x_{F}$-dependence of the three-gluon contribution to $A_{N}$ for $p^{\uparrow} p \rightarrow\left\{\pi^{ \pm, 0}\right.$, jet $\} X$ at $\sqrt{S}=200 \mathrm{GeV}$ and $P_{T}=2 \mathrm{GeV}$. The contribution from $O(x)$ and $N(x)$ are plotted separately for models 1 and 2 .

is probed, and thus the unpolarized gluon density brings main contribution. While the partonic cross sections in the quark fragmentation channel (13)-(15) are tiny, those in the gluon fragmentation channel (17) and (18) for $N(x)$ are large, in particular, the latter contribution is enhanced by the kinematic factor $\hat{s} / \hat{t}$ in (7) for the FSI.

At $x_{F}<0$, in particular, $x_{F} \rightarrow-1$ where $-U \ll S \sim$ $-T$, the region of small- $x$ and large- $x^{\prime}$ is relevant. Thus the model 1 gives rise to only small asymmetry for both $O(x)$ and $N(x)$ due to their mild behavior at small- $x$. On the other hand, the model 2 gives the large asymmetry for the two functions. This is due to the large partonic cross section (8) and (9) in the unpolarized quark distribution 

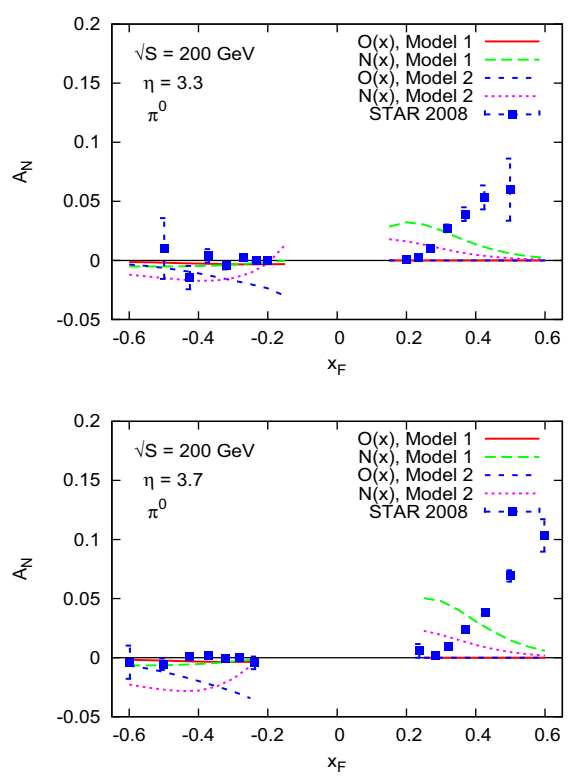

Figure 2. $x_{F}$-dependence of the three-gluon contribution to $A_{N}$ for $p^{\uparrow} p \rightarrow \pi^{0} X$ at $\sqrt{S}=200 \mathrm{GeV}$ and $P_{T}=3.3$ and 3.7 in comparison to the RHIC-STAR data [3]. The contribution from $O(x)$ and $N(x)$ are plotted separately for models 1 and 2 .

channel with the quark fragmentation, and a steeply rising behavior in the three-gluon correlations at small- $x$, which is even more enhanced by the derivative. For $O(x)$, there is a partial cancelation between the ISI and FSI due to the relative signs between (8) and (9), while for $N(x)$ these cross sections contribute constructively. This leads to different behavior of the asymmetry between $N(x)$ and $O(x)$.

Here we note that the three-gluon correlation functions shift the $A_{N}$ for $\pi^{ \pm, 0}$ in the same direction, while the observed asymmetries are approximately $A_{N}^{\pi^{+}} \simeq-A_{N}^{\pi^{-}}$at $x_{F}>0$. This indicates that the three-gluon correlation functions, in particular $N(x)$, cannot be a main source of the $A_{N}$ for the pion.

Recently, AnDY collaboration at RHIC [9] presented a first $A_{N}$ data for the jet production, which shows $A_{N}^{\text {jet }}$ is slightly negative at $x_{F}<0$. Since only the three-gluon correlation function can cause nonzero asymmetry at $x_{F}<$ 0 , the data may be useful to constrain $N(x)$ and $O(x)$.

Figure 2 shows the three-gluon contribution to $A_{N}^{\pi^{0}}$ in comparison to the RHIC-STAR data [3] at $\sqrt{S}=200 \mathrm{GeV}$ and the pseudorapidity $\eta=3.3$ and 3.7. One sees that the contribution from $N(x)$ is much larger than the data at small $x_{F}>0$ for the two models, and therefore it is unlikely that the magnitude of $N(x)$ is as large as these models in the large- $x$ region. At $x_{F}<0$, the contribution of the model 1 is zero and is consistent with data for both $N(x)$ and $O(x)$, while the model 2 for the two functions is far from the data points. This means the three-gluon correlations should behave more mildly than the model 2 in the small- $x$ region. We note, however, that the observed asymmetry results from the combination of the quark-gluon correlation function, twist-3 fragmentation function and the

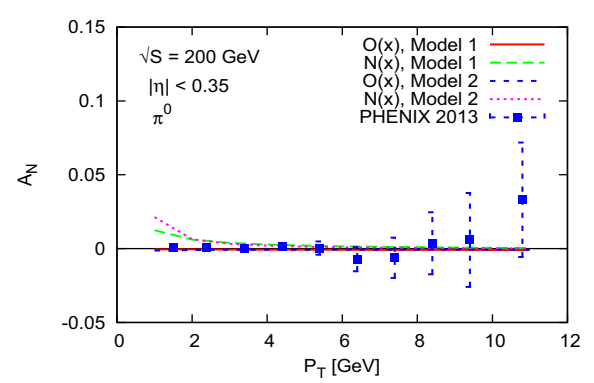

Figure 3. $P_{T}$-dependence of the three-gluon contribution to $A_{N}$ for $p^{\uparrow} p \rightarrow \pi^{0} X$ at $\sqrt{S}=200 \mathrm{GeV}$ and $x_{F}=0$ in comparison to the RHIC-PHENIX data [8]. The contribution from $O(x)$ and $N(x)$ are plotted separately for models 1 and 2 .

three-gluon correlation function, and thus we need a complete analysis including all these effects to draw a definite conclusion.

Figure 3 shows the calculated $A_{N}^{\pi^{0}}$ in the midrapidity region $(|\eta|<0.35)$ at $\sqrt{S}=200 \mathrm{GeV}$ in comparison with the RHIC-PHENIX data [8]. Both models give tiny asymmetry due to the small partonic cross sections, so the form of the three-gluon correlation functions is not much constrained by the data in this region.

\section{Summary}

In this paper we have studied the three-gluon contribution to SSA for the light hadron production in the pp collision, $p^{\uparrow} p \rightarrow h X$. We have derived the corresponding LO twist-3 cross section. Together with the result for the contribution from the quark-gluon correlation and the twist- 3 fragmentation functions, this has completed the twist- 3 cross section for this process. We have also presented a numerical calculation of the asymmetry at the RHIC energy based on our previous models and have shown that this process could bring a useful constraint on the upper bound of the three-gluon correlation functions.

\section{Acknowledgments}

The work of K.K. is supported by the Grand-in-Aid for Scientific Research (No.24.6959) from the Japan Society of Promotion of Science. The work of Y.K. is supported in part by the Grant-in-Aid for Scientific Research (Nos.23540292 and 26287040) from the Japan Society of Promotion of Science. The work of S.Y. is supported by JSPS Strategic Young Researcher Overseas Visits Program for Accelerating Brain Circulation (No.R2411).

\section{References}

[1] J. Adams, et al., STAR Collaboration, Phys. Rev. Lett. 92 (2004) 171801.

[2] S. S. Adler, et al., PHENIX Collaboration, Phys. Rev. Lett. 95 (2005) 202001. 
[3] B. I. Abelev, et al., STAR Collaboration, Phys. Rev. Lett. 101 (2008) 222001.

[4] I. Arsene, et al., BRAHMS Collaboration, Phys. Rev. Lett. 101 (2008) 042001.

[5] L. Adamczyk, et al., STAR Collaboration, Phys. Rev. D 86 (2012) 051101.

[6] L. Adamczyk, et al., STAR Collaboration, Phys. Rev. D 86 (2012) 032006.

[7] L. Adamczyk et al. [STAR Collaboration], arXiv:1309.1800 [nucl-ex].

[8] A. Adare, et al., PHENIX Collaboration, arXiv:1312.1995 [hep-ex].

[9] L. C. Bland et al. [AnDY Collaboration], arXiv:1304.1454 [hep-ex].

[10] J. Qiu, G. Sterman, Nucl. Phys. B 378 (1992) 52.

[11] J. Qiu, G. Sterman, Phys. Rev. D 59 (1998) 014004.

[12] H. Eguchi, Y. Koike, K. Tanaka, Nucl. Phys. B 752 (2006) 1.

[13] H. Eguchi, Y. Koike, and K. Tanaka, Nucl. Phys. B 763, 198 (2007).

[14] C. Kouvaris, J.-W. Qiu, W. Vogelsang, F. Yuan, Phys. Rev. D 74 (2006) 114013.

[15] Y. Koike, K. Tanaka, Phys. Rev. D 76 (2007) 011502.

[16] Y. Koike, T. Tomita, Phys. Lett. B 675 (2009) 181.

[17] A. V. Belitsky, X. D. Ji, W. Lu, J. Osborne, Phys. Rev. D63, 094012 (2001).

[18] V.M. Braun, A.N. Manashov, B. Pirnay, Phys. Rev. D80, 114002 (2009).

[19] H. Beppu, Y. Koike, K. Tanaka, and S. Yoshida, Phys. Rev. D 82, 054005 (2010).

[20] Y. Koike, K. Tanaka, and S. Yoshida, Phys. Rev. D 83, 114014 (2011).
[21] Y. Koike, S. Yoshida, Phys. Rev. D 84 (2011) 014026.

[22] Y. Koike, S. Yoshida, Phys. Rev. D 85 (2012) 034030.

[23] H. Beppu, K. Kanazawa, Y. Koike and S. Yoshida, Phys. Rev. D 89 (2014) 034029.

[24] X. Ji, Phys. Rev. D 49, 114 (1994).

[25] F. Yuan, J. Zhou, Phys. Rev. Lett. 103 (2009) 052001.

[26] Z.-B. Kang, F. Yuan, J. Zhou, Phys. Lett. B 691 (2010) 243.

[27] A. Metz and D. Pitonyak, Phys. Lett. B 723, 365 (2013).

[28] Y. Kanazawa, Y. Koike, Phys. Lett. B 478 (2000) 121.

[29] Y. Kanazawa, Y. Koike, Phys. Lett. B 490 (2000) 99.

[30] K. Kanazawa, Y. Koike, Phys. Rev. D 82 (2010) 034009.

[31] K. Kanazawa, Y. Koike, Phys. Rev. D 83 (2011) 114024.

[32] K. Kanazawa and Y. Koike, Phys. Lett. B 720 (2013) 161.

[33] Z. -B. Kang, J. -W. Qiu, W. Vogelsang and F. Yuan, Phys. Rev. D 83, 094001 (2011).

[34] K. Kanazawa, Y. Koike, A. Metz and D. Pitonyak, Phys. Rev. D 89 (2014) 111501(R).

[35] H. Beppu, Y. Koike, K. Tanaka, S. Yoshida, Phys. Rev. D 85 (2012) 114026.

[36] H. Liu [PHENIX Collaboration], AIP Conf. Proc. 1149 (2009) 439.

[37] M. Gluck, P. Jimenez-Delgado, and E. Reya, Eur. Phys. J. C53 355 (2008).

[38] D. de Florian, R. Sassot, and M. Stratmann, Phys. Rev. D 75, 114010 (2007). 